\title{
Vehicles for Drug Administration to Children: Results and Learnings from an In-Depth Screening of FDA-Recommended Liquids and Soft Foods for Product Quality Assessment
}

\author{
Lisa Freerks ${ }^{1} \cdot$ Wenke Sucher $^{1} \cdot$ Marie-Josefin Tarnow $^{1} \cdot$ Carolin Eckert $^{1} \cdot$ Sandra Klein $^{1}$ (D)
}

Received: 5 January 2022 / Accepted: 17 February 2022 / Published online: 1 March 2022

(c) The Author(s) 2022

\begin{abstract}
Purpose Mixing with liquids or soft foods is a common procedure to improve acceptability of oral medicines in children but may affect drug stability and the in vivo performance of the administered drug product. The aim of the present study was to obtain an overview of the variability of critical attributes of commonly used vehicles and to identify which vehicle characteristics need to be considered when developing in vitro methods for evaluating product quality.

Methods One product of each vehicle listed in the FDA draft guidance "Use of Liquids and/or Soft Foods as Vehicles for Drug Administration" was analyzed with regard to composition, calorific content and physicochemical properties.

Results The studied vehicles show wide variability, both in composition and physicochemical properties. No correlation was observed between vehicle composition and physicochemical properties. Comparison of results of the present study with previously published data also provided variability in physicochemical properties within individual vehicle types.

Conclusions To identify acceptable (qualified) vehicles for global drug product labeling, it is important that the vehicles selected for in vitro compatibility screening reflect the variability in composition and essential physicochemical properties of the vehicles recommended on the product label, rather than relying on results obtained with a single vehicle of each type. Future activities will focus on the development of standardized dosing vehicles that can represent key vehicle characteristics in all their variability to ensure reliable risk assessment.
\end{abstract}

Keywords Bioavailability $\cdot$ Compatibility $\cdot$ Drug stability $\cdot$ Food effect $\cdot$ Pediatric drug product

\section{Introduction}

Despite the growing awareness of the need for age-appropriate formulations, the number of authorized pediatric medicines available is still far behind that of adult medicines. Since there is still a huge need for child-appropriate dosage forms, manipulation of dosage forms is a common practice prior to drug administration to children. Manipulation is defined as the process of physically altering dosage forms in order to provide the prescribed dose and to enhance patient acceptability $[1,2]$. Manipulation of dosage forms can be carried out in different ways depending on the type of the

Sandra Klein

Sandra.Klein@uni-greifswald.de

1 Department of Pharmacy, University of Greifswald, Institute of Biopharmaceutics and Pharmaceutical Technology, Center of Drug Absorption and Transport, Felix Hausdorff Straße 3, 17489 Greifswald, Germany dosage form and the preferences of the caregivers and the pediatric patients, respectively. In the case of solid oral dosage forms, common manipulation practices are for example opening of capsules and splitting or crushing of tablets [1]. Moreover, the manipulated dosage forms as well as other solid oral dosage forms, such as granules or mini tablets, are often mixed with small amounts of soft foods or liquids, in this context referred to as vehicles, prior to drug administration to children with the aim of improving palatability and swallowability $[2,3]$.

So far, there are no precise rules for the joint administration of oral drug products and vehicles. Recommendations regarding suitable vehicles, that become part of the summary of product characteristics (SmPC) and patient information leaflet (PIL) of an individual drug product, are usually made on a product-specific basis. While the suitability of the vehicles listed in SmPCs and PILs has been proven in compatibility studies as part of their authorization and coadministration with them can therefore be considered safe, 
the daily practice of dose administration can be quite different because, as for instance stated by Martir et al., there is no guarantee that parents, caregiver, and healthcare professionals will adhere to the prescribed procedure [4]. Depending on the type of soft food or liquid, dosing vehicles can differ significantly in calorie content, fat:protein:carbohydrate ratios and physicochemical properties. Moreover, even vehicles of the same type can differ due to manufacturing variations related to seasonal, regional, and climatic conditions, or simply by manufacturer and brand [5-8]. In addition, the availability of vehicles, as well as the types of vehicles commonly used can vary significantly around the world [9]. Variations in the type but also the volume of vehicle used to administer the drug product may, however, alter quality and in vivo performance of the drug product. Generally, drugs should be mixed with small volumes $(5-15 \mathrm{~mL})$ of foods or liquids and then administered immediately. A precise upper limit of the maximum permissible vehicle volume has not been specified [10], but it would be essential to prevent unintended in vivo performance of the co-administered drug product.

While the vehicle volumes to be used for administration seem very small at first glance and it would hardly pose a major problem for older children, such as school children and adolescents, whether one administers a dosage form with 5 or $15 \mathrm{~mL}$ of a dosing vehicle, it should be noted that this mode of administration is usually relevant for very young children, i.e., infants and preschool children. If one now assumes a very young infant, for example, administration is made to a patient in which the dimensions of the gastrointestinal tract are still very different from those of a school child or adolescent. Since especially in young infants both gastric capacity and the amount of fasted resting gastric fluid are much smaller than in older children [11], one can imagine that even a small amount of vehicle may be sufficient to alter the gastric environment and to some extent temporarily might also affect intraluminal conditions in the small intestine, increasing the likelihood of changes in the in vivo performance of the drug product. To avoid such undesired effects, only those liquids and/or soft foods that have been shown not to alter the performance of the drug product and which are considered well tolerated and suitable for use in the targeted patient populations should be considered as vehicles for that specific drug product [10]. For some drug products, compatibility with selected vehicles has already been demonstrated [12-20]. On the contrary, there are also some publications which show that vehicles can have a tremendous impact on the drug product performance $[6,7,21-23]$. Given the enormous variety of liquids and soft foods that could potentially be used as dosing vehicles, the question arises as to what potential impact the selected vehicle may have on the stability of the administered drug as well as the in vivo performance of the administered drug product. There is also the question of how best to perform a risk assessment, how many different kinds of vehicles to assess and whether it will be necessary to include different vehicles of the same type in such a study. With the aim of standardizing not only the methodology supporting selection and qualification of the vehicle to be mixed with the drug (product), but also the preparation and use instructions for the drug product vehicle mixture, in 2018 the FDA published the draft guidance "Use of Liquids and/or Soft Foods as Vehicles for Drug Administration: General Considerations for Selection and In Vitro Methods for Product Quality Assessments" [10]. According to this draft guidance only those liquids and/or soft foods demonstrated to have no appreciable effect on drug product performance should be proposed as vehicles and the potential impact of a vehicle on drug product performance should be determined by assessing drug product quality attributes, including potency (assay), in vitro dissolution/release, and other pertinent attributes when the drug product is used with the proposed vehicle(s) [10]. Regarding the selection of vehicles for compatibility assessments, the draft guidance pays particular attention to the $\mathrm{pH}$ value of a potential dosing vehicle. It is stated that "the $\mathrm{pH}$ value of proposed liquids and soft foods should be considered before further testing for their compatibility with the intact or manipulated drug product" [10] and a table of commonly used soft foods and liquids including their approximate $\mathrm{pH}$ range is presented in Appendix A of the draft guidance.

The $\mathrm{pH}$ value of soft foods and fluids is certainly an important parameter, since it can have a major impact on solubility and dissolution rate of ionizable drugs [24]. It can further affect drug stability as well as stability and in vivo performance of the drug product. Particularly for enteric coated drug products, vehicle $\mathrm{pH}$ and mixing time can be very critical, since exposing such formulations to vehicles with higher $\mathrm{pH}$ values could present with the loss of coating integrity which could be accompanied by significant degradation of the drug to be administered [23]. However, it is important to consider whether one should focus on the $\mathrm{pH}$ value in isolation when selecting vehicles for initial compatibility studies, or whether one might want to take a closer look at the composition and other properties that possibly could influence the in vivo performance of the drug product. The reason for such considerations quickly becomes apparent when taking a closer look at Appendix A of the FDA draft guidance, where quite detailed $\mathrm{pH}$ ranges for commonly used liquids and soft foods are listed. If, for example, one now wanted to select a vehicle with a $\mathrm{pH}$ value within a $\mathrm{pH}$ range of 4.4-5.2, the choice would be between buttermilk, yogurt, mashed bananas and maple syrup, i.e., four vehicles for which even without the availability of further detailed information differences in both composition and physicochemical properties can be anticipated. In vitro 
compatibility studies as well as in vivo studies with these vehicles might thus well provide different results despite the same $\mathrm{pH}$ value. Therefore, when it comes to ensuring that the quality of the drug (product) is maintained when mixed with a vehicle and administered to the target patient population, it is advisable to pay attention also to other characteristics besides the $\mathrm{pH}$ of the vehicle from the very beginning. If possible, the detailed composition of the vehicle in terms of fat:protein:carbohydrate ratio, as well as other physicochemical properties, should be considered in order to allow a good pre-selection of vehicles for the assessment of a specific drug product. The goal should be an appropriate risk assessment procedure ensuring the determination of acceptable (qualified) vehicles for drug labeling, but, especially in pediatric drug development, without taking so much time that marketing of the product is unnecessarily delayed.

With the aim of obtaining an initial overview of the variability of the critical attributes of commonly used dosing vehicles and of enabling an estimation of which vehicle properties should be considered when qualifying vehicles for drug labeling, the objective of the present work was to examine one product each of the liquid and soft foods listed in the FDA draft guidance with regard to composition and physicochemical properties.

\section{Materials and Methods}

\section{Materials}

The products investigated in the study are listed in Table 1 . Except for unstrained baby food, one product of each vehicle listed in the FDA draft guidance was examined. The products were purchased from local supermarkets or online shops in various countries such as Germany, the United States (USA), the United Kingdom (UK) and Thailand. All chemicals used for physicochemical characterization were of analytical grade and purchased commercially.

Table 1 Products investigated in the study

\begin{tabular}{|c|c|c|}
\hline Soft food / liquid & Brand mark & Manufacturer \\
\hline Apples (puree) & $\begin{array}{l}\text { Handmade apple puree from } 300 \mathrm{~g} \text { peeled Pink } \\
\text { Lady apples and } 200 \mathrm{~g} \text { demineralized water }\end{array}$ & Pink Lady, Lidl Stiftung \& Co.KG, Neckarsulm, Germany \\
\hline Apple juice & Alosa, Apfelsaft klar & Brands \& Systems BSG GmbH, Hamburg, Germany \\
\hline Applesauce & Babylove, Apfel pur & dm-drogerie markt GmbH + Co. KG, Karlsruhe, Germany \\
\hline Bananas (puree) & Ella's kitchen, bananas & Ella’s kitchen, Henley-on-Thames, UK \\
\hline Buttermilk & Müller, Reine Buttermilch & Molkerei Alois Müller GmbH \& Co KG, Aretsried, Germany \\
\hline Carrots (puree) & dm Bio, Karotte Pur & dm-drogerie markt GmbH + Co. KG, Karlsruhe, Germany \\
\hline Chocolate pudding & Dr. Oetker, Sahne Pudding Vollmilch Schokolade & Dr. August Oetker, Bielefeld, Germany \\
\hline Coconut milk & AROY-D, Coconut Milk & Thai agri Foods Public Company limited, Samutprakarn, Thailand \\
\hline Cranberry juice & Alnavit, Bio Cranberry Muttersaft & Alnavit GmbH, Bickenbach, Germany \\
\hline Drinking water & Humana, Baby-Wasser & Humana Vertriebs GmbH, Bremen, Germany \\
\hline Fruit jelly & Smuckers, Concord Grape Jelly & The J.M. Smucker CO., Orrville, USA \\
\hline Fruit jam & Kroger, Squeezable Jelly Strawberry & Strawberry jam, The Kroger Co., Cincinnati, USA \\
\hline Grapefruit juice & albi, Pink Grapefruit & albi GmbH \& Co. KG, Bühlenhausen, Germany \\
\hline Honey & Langnese, Flotte Biene, Sabienchens Honig & Langnese Honig GmbH \& Co. KG, Bargteheide, Germany \\
\hline Infant formula & Nestlé, Beba Pro 1 Anfangsmilch & Nestlé Nutrition GmbH, Frankfurt, Germany \\
\hline Maple syrup & Tomahawk, Original Kanadischer Ahornsirup & Dockhorn \& Co. Import-Export GmbH, Hamburg, Germany \\
\hline Milk & Weihenstephan, Haltbare Milch 3.5\% Fett & Molkerei Weihenstephan GmbH \& Co. KG, Freising, Germany \\
\hline Orange juice & Hohes C, Orange & Eckes-Granini Deutschland GmbH, Nieder-Olm, Germany \\
\hline Orange marmalade & Valensina, Orange, Fein passiert & Zentis GmbH \& Co KG, Aachen, Germany \\
\hline Peanut butter & Nick, Peanut-butter creamy & Rila Feinkost-Importe GmbH \& Co. KG, Stemwede-Levern, Germany \\
\hline Pineapple juice & Edeka, Ananas Direkt Saft! & Edeka Zentrale Stiftung \& Co. KG, Hamburg, Germany \\
\hline Rice pudding & Ambrosia, Rice Pudding & The Premier Foods Group, London, UK \\
\hline Soybean milk & dm Bio, Soja Drink Natur & dm-drogerie markt GmbH + Co. KG, Karlsruhe, Germany \\
\hline Strawberries & $\begin{array}{l}\text { Rewe Beste Wahl Erdbeeren, } \\
\text { Ganze Früchte tiefgefroren }\end{array}$ & Rewe "Beste Wahl", Köln, Germany \\
\hline Yogurt & $\begin{array}{l}\text { Nestlé, LC1 Pur, Joghurt mild } \\
3.5 \% \text { Fett }\end{array}$ & Lactalis Nestlé Frischprodukte Deutschland GmbH, Kehl, Germany \\
\hline
\end{tabular}




\section{Preparation of the vehicles prior to physicochemical characterization}

Most of the vehicles listed in the FDA draft guidance are commercially available liquid or semisolid foods characterized by a more or less homogeneous state with varying consistencies. Except for apple puree and strawberries, commercially available soft foods and liquids were studied. Apple puree was prepared from fresh apples. For this purpose, $300 \mathrm{~g}$ of peeled and sliced apples and $200 \mathrm{~g}$ of demineralized water were placed in a 1-L beaker and heated on a hot plate with occasional stirring (IKA ${ }^{\circledR}$ RCT basic, IKA-Werke GmbH \& Co. KG, Staufen, Germany), first to $60{ }^{\circ} \mathrm{C}$ for $15 \mathrm{~min}$ and then to $80{ }^{\circ} \mathrm{C}$ for another $10 \mathrm{~min}$. The mixture was subsequently homogenized with a stick blender (P8-RM-SB, Dirk Rossmann GmbH, Burgwedel, Germany) for $30 \mathrm{~s}$. To investigate the physicochemical properties of strawberries, frozen strawberries were thawed to room temperature and homogenized with a stick blender for $30 \mathrm{~s}$.

\section{Composition of the vehicles investigated in the study}

Specific product information, such as the fat:protein:carbohydrate ratio and calorific content of the individual vehicles, was taken from the product labels and documented for further evaluation. Since the apples used to prepare the apple puree were not labeled, the detailed product information was taken from the website of the producer [25].

\section{Physicochemical characterization of the vehicles}

The physicochemical properties, i.e., the density, $\mathrm{pH}$, buffer capacity, osmolality, surface tension and the viscosity/ rheological properties of all vehicles listed in Table 1 were measured as described in [5] if not stated otherwise. With the exception of rheological measurements $(n=3)$, all tests were performed in sextuplicate $(n=6)$, and the results were expressed as mean \pm standard deviation (S.D.). Except for osmolality, parameters were recorded at $25.0{ }^{\circ} \mathrm{C} \pm 0.5^{\circ} \mathrm{C}$.

\section{Density}

The density of fluids was measured using a digital density meter (Type DMA 10, serial no: 1030, Anton Paar GmbH, Graz, Austria). The density of semisolid foods was determined using a $10 \mathrm{~mL}$ measuring cylinder by weighing the measuring cylinder on an analytical balance (MC1 Laboratory LC 620 P, Sartorius AG, Göttingen, Germany) before and after adding $10 \mathrm{~mL}$ of vehicle. The densities of the semisolid foods were used to calculate the accurate vehicle masses required for the experiments described in the following sections.

\section{$\mathrm{pH}$ value and buffer capacity}

The $\mathrm{pH}$ value was measured using a calibrated $\mathrm{pH}$-meter (HI 99,161, with electrode FC202D, HANNA Instruments Deutschland GmbH, Vöhringen, Germany). In order to determine the buffer capacity of fluids, defined volumes were tempered in a laboratory shaker $\left(\mathrm{IKA}^{\circledR} \mathrm{KS} 3000 \mathrm{i}\right.$ control, IKA-Werke GmbH \& Co. KG, Staufen, Germany) and quantified by potentiometric titration using different concentrations of hydrochloric acid $(0.01 \mathrm{M}, 0.02 \mathrm{M}, 0.03 \mathrm{M}$, $0.05 \mathrm{M}, 0.1 \mathrm{M}$ or $0.2 \mathrm{M} \mathrm{HCl}$, Merck KGaA, Darmstadt, Germany). For measuring the buffer capacity of the soft foods, a defined mass was mixed with demineralized water and then titrated as described for the fluids. For calculating the buffer capacity according to USP, the volumes of hydrochloric acid were determined which resulted in a change of the $\mathrm{pH}$ value by one unit.

\section{Osmolality}

The osmolality was measured via freezing point depression method (semi-micro osmometer K-7400, Knauer Wissenschaftliche Geräte GmbH, Berlin, Germany). Where possible, osmolality of the vehicles was measured directly without any further preparatory treatment. Since the osmolality of semisolid foods and some of the fluids could not be assessed directly, a set of four dilutions was prepared with demineralized water. These dilutions were mixed for $1 \mathrm{~min}$ using a Vortex mixer (VWR Reagenzglasschuettler, VWR International $\mathrm{GmbH}$, Darmstadt, Germany) and then centrifuged for $15 \mathrm{~min}$ at 4,000 rpm (Eppendorf Centrifuge 5702 R, Eppendorf AG, Hamburg, Germany). $150 \mu \mathrm{L}$ of the aqueous phase were then added to a $1.5 \mathrm{~mL}$ SafeSeal reaction tube (Sarstedt AG \& Co. KG, Nürnbrecht, Germany) and each dilution was measured six times. Since a positive correlation between the dilution and the measured osmolality was observed (regression coefficient $\left(R^{2}\right)>0.97$ for maple syrup and $R^{2} \geq 0.99$ for all other vehicles requiring dilution), the osmolality of the undiluted vehicle was extrapolated via linear regression.

\section{Surface tension}

The surface tension of the vehicles was determined with a ring tensiometer (K11, Krüss GmbH, Hamburg, Germany). For determining the surface tension of semisolid foods, a set of four different aqueous dilutions was prepared. Each dilution was measured in sextuplicate. Since there was no big change in the surface activity of the different dilutions, 
it was assumed that surfactant concentrations were above the critical micelle concentration (CMC) and the surface tension of the aqueous phase of the vehicles was calculated as the mean \pm standard deviation of all measured values $(n=24)$.

\section{Viscosity}

The viscosity of all Newtonian fluids was determined with a suitable Ubbelohde viscosimeter (either type 0c, $\mathrm{K}=0.003188 \mathrm{~mm}^{2} / \mathrm{s}^{2}$; type $\mathrm{I}, \mathrm{K}=0.009623 \mathrm{~mm}^{2} / \mathrm{s}^{2}$ and $\mathrm{K}=0.010080 \mathrm{~mm}^{2} / \mathrm{s}^{2}$; or type II, $\mathrm{K}=0.099370 \mathrm{~mm}^{2} / \mathrm{s}^{2}$; calibrated according to DIN 51,562; SI Analytics, Mainz, Germany).

Due to the fruit pulp present in pineapple juice and the high viscosity and non-Newtonian flow behavior of the semi-solid foods, the rheological properties of these vehicles were determined using a cup and bob rotational viscometer (Brookfield DV3T with DIN 87 spindle and ULA-DIN-6Y sample container, Brookfield Engineering Laboratories, Middleborough, USA) applying various shear rates.

\section{Results and Discussion}

With the goal of gaining better insight into the variability of critical attributes of commonly used dosing vehicles, one representative of each of the vehicles listed in the FDA draft guidance was thoroughly investigated. Although grapefruit juice is not recommended as a vehicle at all and honey is not recommended for children under the age of 12 , they were included in the study, since dosing vehicles are not just used in pediatric patients, but also other patient populations who are unable to swallow solid oral dosage forms. Results on composition and physicochemical properties of the vehicles listed in Appendix A, which are presented and discussed in the following section will therefore be of interest for nonpediatric applications as well.

\section{Composition of vehicles investigated in the study}

The vehicle composition is an important factor to consider when co-administering a drug product with food or fluids as it can influence stability, solubility, and dissolution rate of drugs. The extent to which the dosing vehicle affects one or more of these parameters depends on many factors, including the drug substance and its formulation, the composition and physicochemical properties of the vehicle, the contact time with the vehicle prior to ingestion, the amount of dosing vehicle used to administer the dosage form and, the physiological conditions in the patient's gastrointestinal tract. The latter in turn depend very much on the patient's age, size, body weight, and possibly also on the patient's disease status. When co-administered with vehicles with a high fat content, poorly water-soluble lipophilic drugs may (partly) dissolve in components of the dosing vehicle, whereas in pure water, they often may not dissolve at all [24, 26]. This could be associated with altered bioavailability, especially in very young children in which the resting volumes of gastric and small intestinal fluid are still relatively small and thus the volume and composition of the co-administered vehicle may have, at least temporarily, a much stronger impact on intraluminal conditions than in older children [11].

However, vehicles can influence the solubilization of drugs not only directly but also indirectly. For example, during digestion the distribution of drugs between the lipid phase and the aqueous phase of the gastrointestinal contents may be altered, which might also influence drug absorption. Such effects would also be expected primarily for lipophilic drugs $[27,28]$. In addition to altering intraluminal contents, the co-administered vehicle may have further effects on physiological parameters, such as for instance gastric emptying rate. After food ingestion one of the factors determining gastric emptying rate is the caloric content of the food ingested. Increasing caloric content of the co-administered food or liquid may lead to an increase in the residence time of drugs in the stomach, possibly resulting in a delay in drug absorption [26]. As mentioned before, fasted administration of small amounts (e.g., $5 \mathrm{~mL}$ ) of dosing vehicles in older children will most likely not result in pronounced vehicle-related food effects, whereas, when co-administering somewhat larger vehicle volumes in very young children, the possibility of such effects should certainly be kept in mind.

Figure 1 represents the fat-, carbohydrate- and proteincontent as well as the calorific content per $100 \mathrm{~mL}$ of fluid or per $100 \mathrm{~g}$ of semisolid food, respectively. Most of the vehicles studied are carbohydrate-based, but overall, there is wide variability in both the percentages of fat, carbohydrates, and proteins as well as in the absolute nutritional values of the vehicles. Coconut milk (19\% fat) and peanut butter ( $47 \%$ fat), for example, are vehicles with significantly higher fat contents, which should be considered when coadministering these vehicles with lipophilic drugs.

\section{Physicochemical properties of vehicles investigated in the study}

Each of the physicochemical properties assessed in this study may have an impact on drug stability and drug product performance. The influence of the $\mathrm{pH}$ value on the solubility and dissolution rate of ionizable drugs as well as the integrity of enteric coatings has already been mentioned. The $\mathrm{pH}$ conditions in the gastrointestinal lumen are subject to a wide variety of factors. If a drug is co-administered with a dosing vehicle in the fasted state, $\mathrm{pH}$ and buffering capacity of the co-administered vehicle may have a significant impact on the intraluminal $\mathrm{pH}$ conditions, especially in very 
Fig. 1 Fat:carbohydrate:prote in ratio and calorific content of the vehicles investigated in the study macronutrients

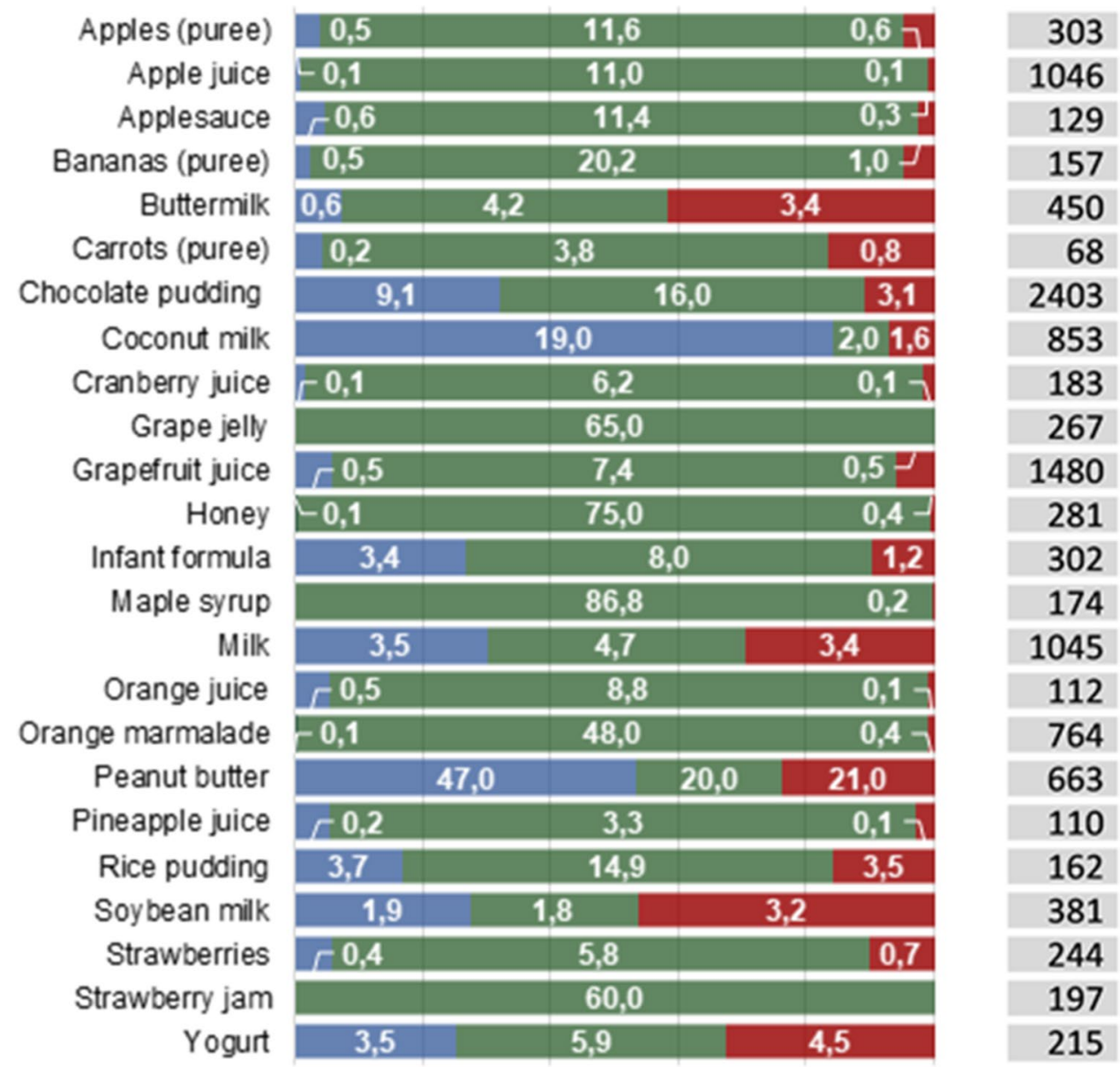

= fat in g per $100 \mathrm{~mL} 100 \mathrm{~g}$

- carbohydrates in g per $100 \mathrm{~mL} 100 \mathrm{~g}$

- protein in g per $100 \mathrm{~mL} 100 \mathrm{~g}$

- calories in $\mathrm{kJ}$ per $100 \mathrm{~mL} 100 \mathrm{~g}$ young children. The surface tension of the vehicle can affect drug release/dissolution, as it affects wetting and thus the effective surface area of the drug available for dissolution and, depending on the CMC of the surfactant present in the vehicle, there might even be a chance for increased drug solubilization immediately after administration. Therefore, vehicles with a low surface tension may contribute to the dissolution and, in individual cases, possibly also the bioavailability of poorly soluble drugs. Although this might be of only minor impact in most cases, vehicle osmolality, by contrast, may impact drug release of dosage forms that release the drug depending on osmotic pressure differences [24]. A high vehicle viscosity as well as an increase in viscosity of luminal contents has proven to negatively affect the dissolution of various drugs as it results in a decreased diffusivity $[7,24]$. Moreover, vehicles that contribute to a higher osmolality and/or viscosity of the gastric contents could also affect gastric emptying which is, however, controlled by several additional factors including the caloric content and the detailed composition of gastric contents [29-32]. Based on these considerations, it is obvious that the potential impact of vehicles on rate and extent of oral drug absorption goes far beyond the impact of the vehicle $\mathrm{pH}$, which is why essential differences of typical vehicles were studied in more detail. The physicochemical properties of the vehicles investigated in this study are summarized in Figs. 2, 3 and 4.

Appendix A of the FDA draft guidance provides approximate $\mathrm{pH}$ ranges of the different soft foods and liquids regarded as commonly used vehicles. Since many of the listed values are given very precisely, it looks at first glance as if this information is very reliable. At second glance, however, one notices that often only one value is specified 

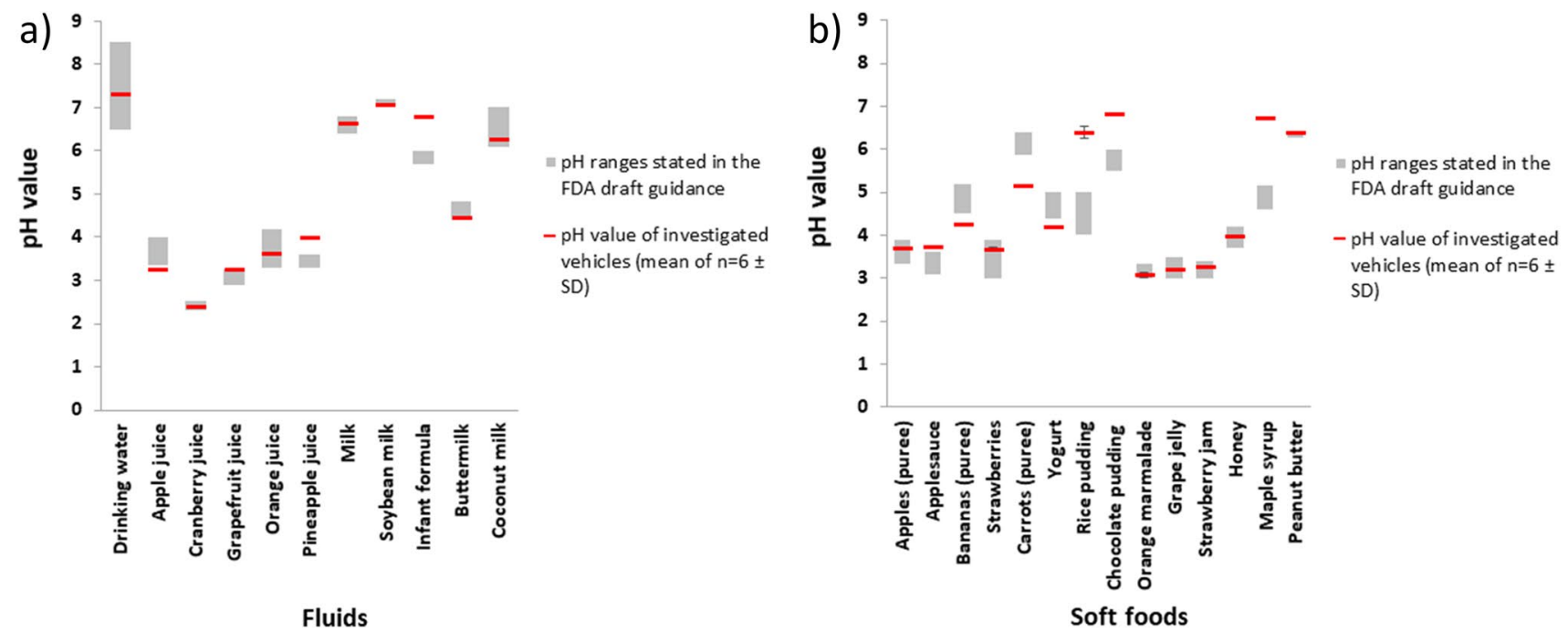

Fig. $2 \mathrm{pH}$ values of the vehicles investigated in the study (mean of $\mathrm{n}=6 \pm$ S.D.) in comparison to the $\mathrm{pH}$ ranges specified in Appendix A of the FDA draft guidance [10]

with standard deviation or minimum and maximum values being missing, which seems very unlikely given the large variety of food products available. If then following the link provided, which leads to the source of the majority of these data (https://hgic.clemson.edu/factsheet/canni ng-foods-the-ph-factor/), it turns out that this is a fact sheet specifying how food should be processed for preservation depending on its $\mathrm{pH}$. Since this is anything but a scientific publication, the true origin of the data remains unknown. To determine whether the $\mathrm{pH}$ ranges given in Appendix $\mathrm{A}$ are representative, the $\mathrm{pH}$ of one product/preparation of each of the listed vehicles was measured and compared to the $\mathrm{pH}$ (range) reported in Appendix A (Fig. 2). Results shown in Fig. 2 confirmed that most of the vehicle $\mathrm{pH}$ values are in the acidic range but indicate that the experimentally determined $\mathrm{pH}$ values are partly within the indicated ranges, whereas for some vehicles, such as for instance infant formula, maple syrup, rice pudding etc. the $\mathrm{pH}$ can be far outside that range, indicating a high variability of $\mathrm{pH}$ values even within the same vehicle type. Already after determining the $\mathrm{pH}$ of a single vehicle of each type, it is therefore clear that the $\mathrm{pH}$ data from Appendix A cannot be regarded as scientifically sound, which is probably also the reason why the draft guidance states that these are approximate $\mathrm{pH}$ ranges. It is likely that also the $\mathrm{pH}$ ranges provided here were measured for just one or very few representatives of each vehicle type. The results shown in Fig. 2 clearly indicate that the $\mathrm{pH}$ of the vehicle may be subject to considerable variability for various reasons. While the $\mathrm{pH}$ of unprocessed foods and liquids can vary depending on variety, origin, and season, it is quite common to adjust the $\mathrm{pH}$ of processed foods to optimize the chemical stability of the vehicle as well as other key vehicle properties such as taste, viscosity, and texture. Regardless of the reasons for which the $\mathrm{pH}$ may vary, this variability must be taken into account when specifying acceptable (qualified) dosage vehicles in drug product labeling. Of some vehicles, many different subtypes and brands are available, which differ considerably in composition and possibly also in physicochemical properties. Nevertheless, they are often grouped under a collective name. This applies in particular to milk, infant formula, and unstrained baby food, but also to many other liquids and soft foods. In the case of unstrained baby food, no specific food product was tested in the present study, because this general name could not be clearly assigned to a specific kind of vehicle. It is therefore even more interesting that in Appendix A a very narrow $\mathrm{pH}$ range of $\mathrm{pH} 5.95$ to 6.05 is specified for unstrained baby food. With regard to the stated $\mathrm{pH}$ value, two other vehicles (peanut butter and soy milk) were initially conspicuous, as there is no $\mathrm{pH}$ range stated for either of them, but fixed $\mathrm{pH}$ values of $\mathrm{pH} 6.28$ for peanut butter and $\mathrm{pH} 7$ for soy milk, respectively. The $\mathrm{pH}$ measurement in the present study did indeed provide similar values with a measured $\mathrm{pH}$ value of 6.41 for peanut butter and $\mathrm{pH} 7.06$ for soy milk for the products assessed. Whether this is a random incident or whether the $\mathrm{pH}$ value of these vehicles is generally subject to only small fluctuations would need to be clarified in further investigations. Overall, results of the $\mathrm{pH}$ measurements raise the question of how many vehicles of the same type should be studied to determine adequate and comprehensive ranges of $\mathrm{pH}$ values which would be essential for proper risk assessment in the process of identifying acceptable (qualified) vehicles for labeling.

In addition to $\mathrm{pH}$, several other characteristics of the vehicle can affect drug stability and drug product performance. For this reason, buffer capacity, osmolality, surface tension, 
Fig. 3 Buffer capacity (a), osmolality (b), surface tension (c) and viscosity (d) of the vehicles investigated in the study (mean of $n=6 \pm$ S.D.)
Fluids

a)

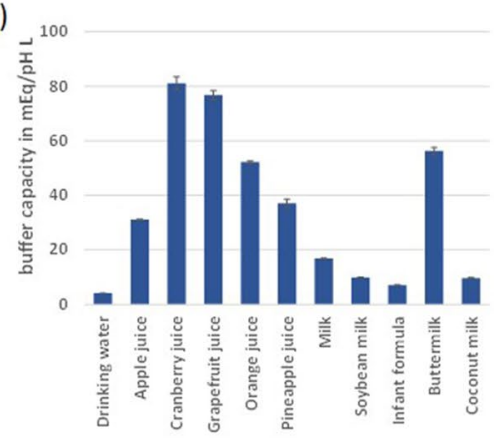

Fluids

b)

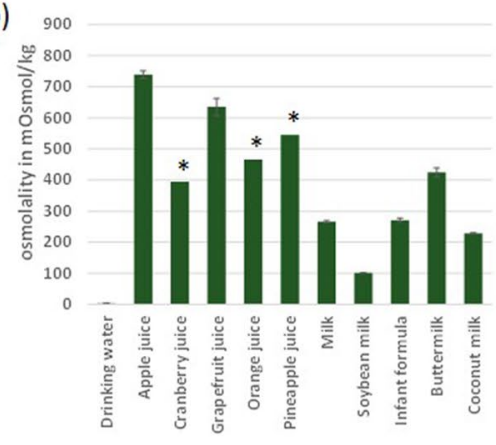

Fluids

c)

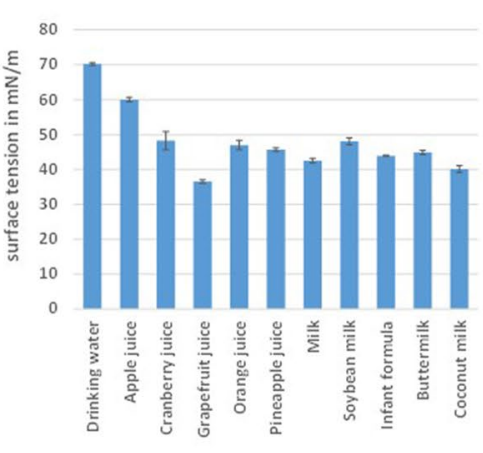

Fluids

d)

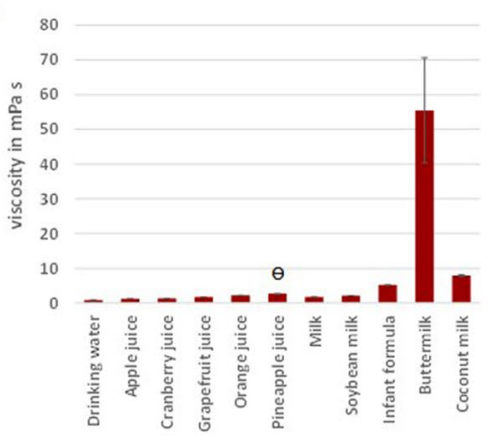

Soft foods

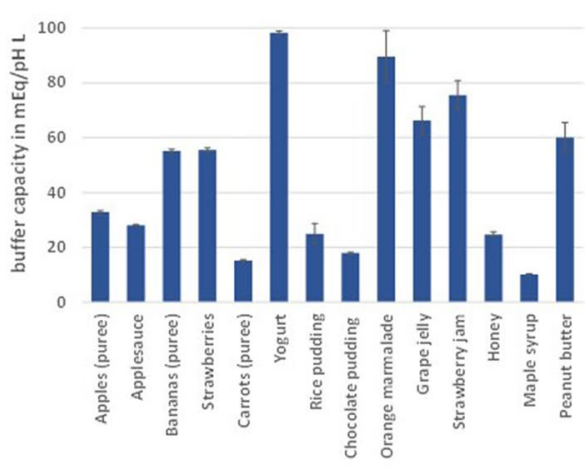

Soft foods

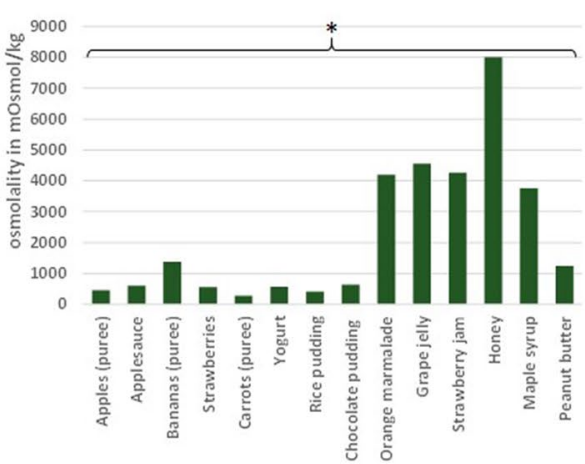

Soft foods

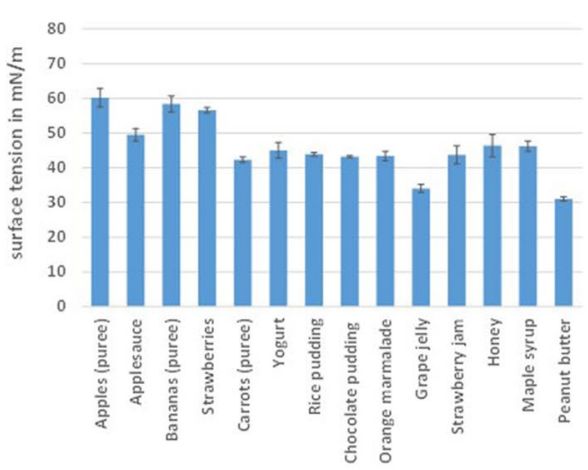

Soft foods

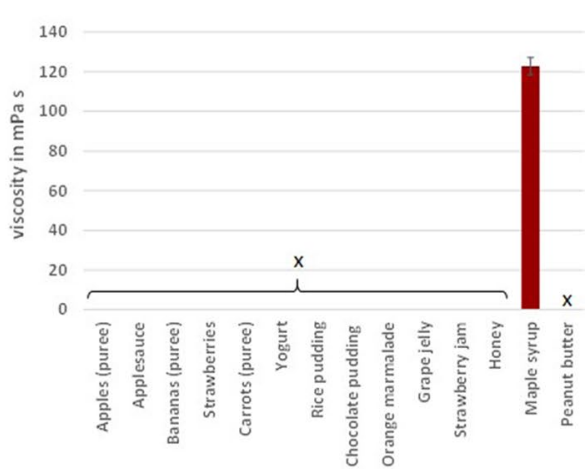

* the osmolalities were obtained by extrapolation and therefore no standard deviation is shown - the viscosity was measured via rotational viscosimeter and showed Newtonian flow behavior $\mathrm{x}$ the viscosity was measured via rotational viscometer 

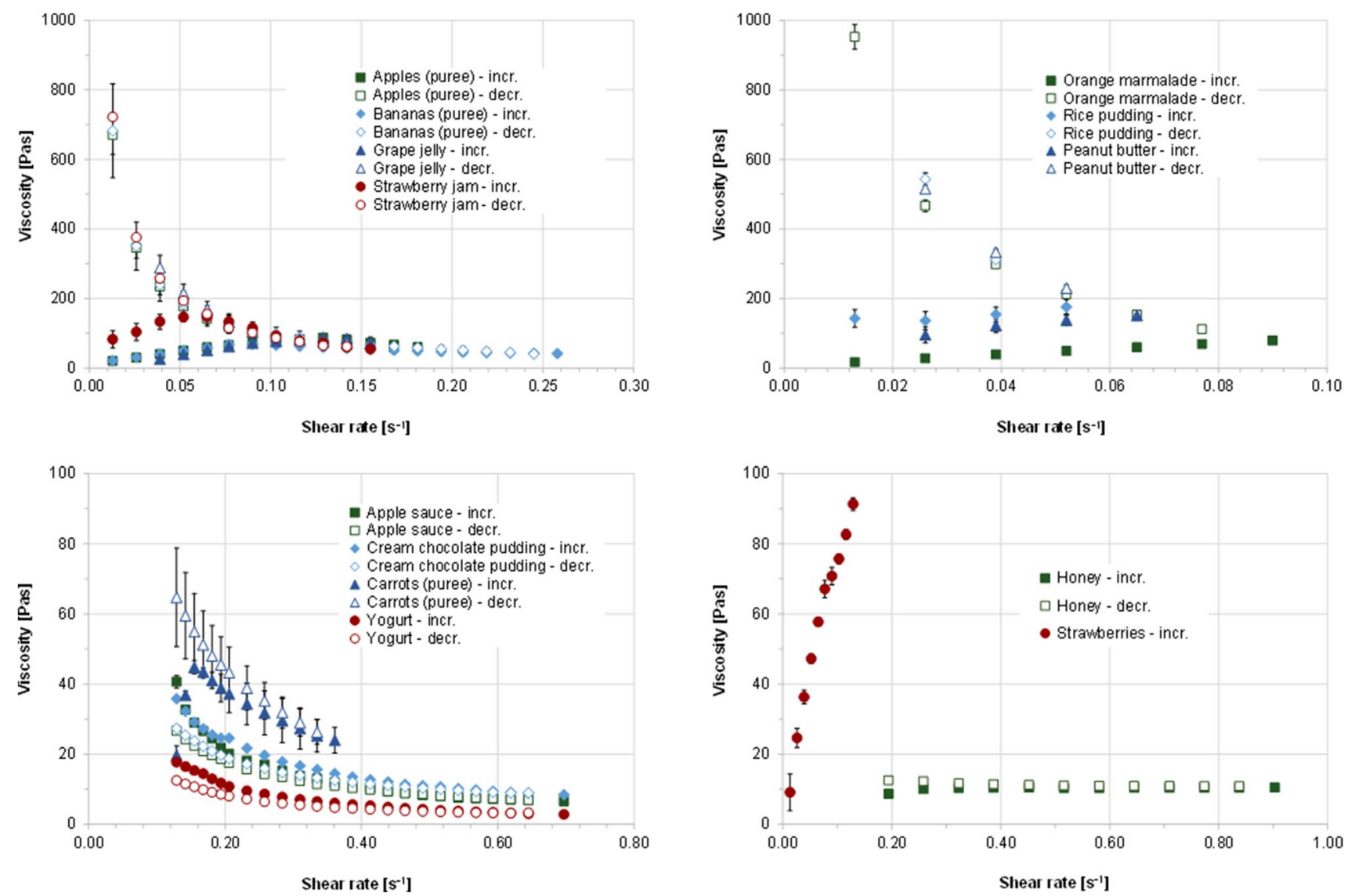

Fig. 4 Viscosity profiles of investigated soft foods at $25^{\circ} \mathrm{C}$ and increasing and decreasing shear rates (mean of $n=3 \pm$ S.D.)

and viscosity/rheological properties were also investigated. Density is not discussed in detail here, as it was mainly used to calculate food volumes based on the masses of the soft foods. An overview of the properties of all vehicles, grouped into fluids and soft foods, is shown in Fig. 3. Since most of the soft foods either showed non-Newtonian flow behavior or their viscosity was too high for using an Ubbelohde viscosimeter, their flow behavior was measured with a rotational viscometer and is depicted in Fig. 4.

For all physicochemical properties investigated, large differences could be observed among the vehicle types. Overall, surface tension was the parameter with the smallest fluctuations, while strong variability was observed for the other parameters. Expectedly, buffer capacity was lowest for drinking water $(0.11 \pm 0.00 \mathrm{mEq} / \mathrm{pH} / \mathrm{L})$. The highest buffer capacity was measured for yogurt $(98.27 \pm 0.65 \mathrm{mEq} / \mathrm{pH} / \mathrm{L})$, which contains a relatively balanced mix of fat, carbohydrates and proteins, followed by orange marmalade $(89.50 \pm 9.43 \mathrm{mEq} /$ $\mathrm{pH} / \mathrm{L})$, cranberry juice $(81.13 \pm 2.42 \mathrm{mEq} / \mathrm{pH} / \mathrm{L})$, grapefruit juice $(76.75 \pm 1.64 \mathrm{mEq} / \mathrm{pH} / \mathrm{L})$, strawberry jam $(75.50 \pm 5.24 \mathrm{mEq} / \mathrm{pH} / \mathrm{L})$ and grape jelly $(66.25 \pm 5.07 \mathrm{mEq} /$ $\mathrm{pH} / \mathrm{L}$ ), all of which are very high in carbohydrates, particularly mono- and disaccharides. If one also considers the buffer capacities of the other soft foods and liquids, it becomes apparent that there is no real correlation between the content and ratio of macronutrients and the buffer capacity. Here, it would be necessary to look at the composition of each vehicle down to the smallest detail. As for the buffer capacity, osmolality was lowest for drinking water $(4 \pm 1$ $\mathrm{mOsmol} / \mathrm{kg}$ ), since a drinking water obtained by reverse osmosis was assessed in the study. Soft foods rich in shortchain carbohydrates, such as honey (8005 mOsmol/ $\mathrm{kg}$ ), grape jelly ( $4555 \mathrm{mOsmol} / \mathrm{kg})$, strawberry jam (4267 mOs$\mathrm{mol} / \mathrm{kg}$ ), orange marmalade $(4201 \mathrm{mOsmol} / \mathrm{kg}$ ) and maple syrup (3758 mOsmol $/ \mathrm{kg}$ ) had the highest osmolalities, which for the most part were close to 10 times higher than those of the liquids and most of the other soft foods. At this point, it should be briefly noted that the approach of measuring a dilution series of the vehicle to extrapolate the osmolality of the undiluted vehicle may possibly lead to a slight overestimation of the osmotic activity of the undiluted preparation, since one creates optimal conditions for the complete dissociation of osmotically active particles by dilution. However, such theoretically possible variations have little effect on the large osmolality differences between soft foods rich in shortchain carbohydrates and other vehicles. It should also be noted that after oral administration, the vehicles mix with the co-administered liquid (usually water) and gastrointestinal 
secretions and are thereby diluted, which, as shown by the dilution series results, allows for complete dissociation, so the discussion at this point is probably just of theoretical nature.

Surface tension was lowest for peanut butter $(31.02 \pm 0.60$ $\mathrm{mN} / \mathrm{m}$ ), representing the vehicle with the highest fat content, followed by grape jelly $(34.09 \pm 1.11 \mathrm{mN} / \mathrm{m})$ and grapefruit juice $(36.50 \pm 0.48 \mathrm{mN} / \mathrm{m})$, which are rich in sugar, and coconut milk $(40.09 \pm 1.00 \mathrm{mN} / \mathrm{m})$, which again has a high fat content. As for the buffer capacity, there was no direct link between the content and ratio of macronutrients and the surface tension and more detailed information on the composition would be required to possibly derive influences on the surface tension.

While the buffer capacities and osmolalities of some vehicles differed by as much as a factor of ten, the differences in viscosities were even greater. The rheological properties of the soft foods also varied, with most vehicles exhibiting either shear thinning or more Newtonian flow behavior as the shear rate increased. Some vehicles, particularly the purees, exhibited slightly dilatant flow behavior at the onset of the measurements, likely due to food particles that initially had to orient themselves in the shear direction. Dilatant flow behavior was seen particularly for orange marmalade, peanut butter, and strawberries, which can be attributed to a high content of food particles. This is probably also the reason why some vehicles showed rheopex flow behavior with increasing viscosity as a function of time of shear stress. In the case of strawberries, this behavior was so pronounced that it was not possible to measure the viscosity profile at decreasing shear rates. However, the pronounced dilatant flow behavior of the pureed strawberries was most likely due to the gap geometry of the rheometer. It can be assumed that strawberry seeds have wedged themselves here in a way which is very unlikely to be observed in vivo. Overall, results obtained when assessing the flow behavior indicate that when a sufficient shear force is applied most of the semisolid vehicles can freely flow. It might be speculative to generally conclude that following dose administration with a semisolid dosing vehicle the shear forces caused by gut motility as well as in vivo dilution and digestion of the vehicle will ensure that the drug product is not "entrapped" in the dosing vehicle for a longer time period, but the probability for such an observation seems rather low, since particularly in combination these effects are likely to significantly reduce the vehicle viscosity. The age group for which there is probably the greatest risk for an "entrapment" effect caused by highly viscous vehicles is again the cohort of very young infants, for which, however, one would not as a rule already co-administer medications with semi-solid-, but rather with liquid vehicles. However, if one considers that the drug is mixed with the vehicle prior to administration and that administration does not always take place immediately after mixing as foreseen, then viscosity may well have an influence on the stability and the in vivo performance of the dosage form. Low-viscosity liquids can certainly penetrate the dosage form and interact with the drug much more rapidly than the free liquid phase in semi-solid vehicles. However, such effects must be discussed on an individual basis, since several other factors, such as the type of drug product, the composition of the vehicle and possibly other physicochemical properties of the vehicle, are of course also important in this context.

Interestingly, no correlation was observed between vehicle composition and its physicochemical properties. Vehicles belonging to one category of food often differ significantly in their physicochemical properties, as can be seen, for example, in the results for milk and yogurt, both of which belong to the dairy products group. Since the goal of the study was to provide an overview of essential differences in composition and properties of "randomly" selected vehicles of each type, but not to examine variability in composition and properties of individual vehicles, such data are not shown here. However, there are already a few publications that address the properties of further brands of the vehicles listed in Appendix A [5, $6,13]$. Comparing the results of this study with previously published values, variability in physicochemical properties can also be observed within one vehicle type. As discussed earlier, the amount of vehicle used for co-administration and the age of the pediatric patients certainly also determine the extent to which the different properties of the vehicles affect drug stability and in vivo performance of different dosage forms. At least for certain dosage form-vehicle combinations, an increasing impact can be expected with very young patient age and with increases in vehicle volume and/or exposure time.

\section{Current and proposed methodology supporting vehicle selection}

The differences in the composition and physicochemical properties of commonly used vehicles reported in this work are important information when estimating the compatibility of pediatric drugs with soft foods and liquids. From all the studies conducted so far, we have learned that in compatibility assessment it is important to look at the composition and physicochemical properties of a vehicle as whole and not to focus on a single parameter, such as $\mathrm{pH}$. In the present study, one commercially available liquid or soft food was investigated as an exemplary vehicle for each of the vehicles listed in Appendix A of the FDA draft guidance to estimate essential differences between the individual vehicles and how physicochemical properties may be linked to their composition. It can however be assumed that composition and physicochemical properties 
not just vary among different vehicles, but also within one vehicle type, which has already been indicated by the results of some preliminary experimental work. Moreover, even vehicles of the same brand may have different compositions in different countries. Therefore, especially when developing drugs to be approved in many countries around the world, one should not disregard the global availability and the quality of the vehicles listed in the label, since this might pose a fundamental problem regarding a reliable risk assessment. On the other hand, although desirable, it will be impossible to realize the statement in FDA's draft guidance "Assessing the Effects of Food on Drugs in INDs and NDAs-Clinical Pharmacology Considerations" that "all soft foods intended for labeling should be tested" [33]. Compatibility testing with all potential dosing vehicles while considering the variability of each individual vehicle would be a tremendous burden for the sponsor. Moreover, such an effort, which is certainly not feasible, could only be effective if the variability of each vehicle would be known. Nevertheless, a well-designed in vitro compatibility test is considered an important tool for assessing product quality, since it will help to reduce the number of in vivo studies required for risk assessment and should therefore be implemented by all means. The current FDA draft guidance aims to ensure consistent quality of the drug when administered with a vehicle and indicates the need to standardize the methodology for vehicle selection as well as the preparation and use instructions for the drugvehicle mixture. However, the focus here is always on one vehicle type, e.g., apple sauce, pudding, etc., without considering how variable its properties can be. Thus, conducting compatibility studies without knowing whether the vehicle chosen for these studies is representative of all vehicles of this type may pose a serious risk. Based on the discussion so far, a better understanding of the variability of individual vehicles should first be sought so that in future compatibility studies a limited number of standardized vehicles which clearly reflect the variability in vehicle composition and physicochemical properties that may be critical to the stability of the drug and the in vivo performance of the drug product in question can be used. Critical quality attributes that should be considered are the physicochemical properties highlighted in the present study, i.e. $\mathrm{pH}$, buffer capacity, surface tension, osmolality, viscosity but also the composition of the vehicle, including the carbohydrate:fat:protein ratio, and the calorific content. Depending on the drug under investigation, other vehicle properties such as the presence of metal ions, oxidizing agents and other ingredients that could catalyze drug degradation may also be important. To take all these factors into account in a general test design would certainly go beyond the scope. However, they might be taken into account in product-specific studies if the drug product to be administered contains a drug substance for which such specific stability problems are already known from early development phases. With the aim of establishing a toolbox of standard vehicles that will allow an appropriate risk assessment of co-administering pediatric dosage forms with soft foods and liquids in different locations around the world, the experimental approach of detailed characterization of soft foods and liquids presented here will be pursued and specifically continued with a larger number of representatives for each vehicle type.

\section{Conclusion}

When selecting a vehicle for the administration of oral dosage forms to children, $\mathrm{pH}$ is often the most important criterion for ensuring compatibility of drug substance or drug product and vehicle. Results of the investigation of the composition and physicochemical properties of one of each of the vehicles listed in the FDA draft guidance document "Use of Liquids and/or Soft Foods as Vehicles for Drug Administration", indicate that $\mathrm{pH}$ is an important, but not the only parameter that should be considered when evaluating the compatibility of liquids and soft foods used as vehicles for drug administration to children. Vehicles with similar $\mathrm{pH}$ can significantly differ in composition and in other physicochemical properties that, when co-administered with oral dosage forms, could impact drug product quality. Moreover, even the $\mathrm{pH}$ of individual vehicles can be far more variable than indicated in the draft guidance. Compatibility assessment performed with a certain brand of soft food or liquid will thus not necessarily be predictive for the whole range of marketed products of the same food/fluid type. To identify acceptable (qualified) vehicles for drug product labeling, it is important that the vehicles selected for in vitro compatibility screening reflect the variability in composition and essential physicochemical properties of the vehicles recommended on the product label, rather than relying on results obtained with a single vehicle of each type. Furthermore, in order to make the procedure more meaningful with regard to the administration of the drug under investigation to pediatric- as well as other patients around the world and to provide reliable and robust data to both the pharmaceutical developer and regulatory authorities, an appropriate standardization of the in vitro method should be considered.

Acknowledgements Part of the research described in this paper was sponsored by a Ph.D. grant from Janssen Research \& Development, A Division of Janssen Pharmaceutica NV. Our special thanks in conjunction with this support go to Tina Arien, Sabine Inghelbrecht and Claire Mackie.

Funding Open Access funding enabled and organized by Projekt DEAL. 


\section{Declarations}

Competing Interests The authors declare that they have no known competing financial interests or personal relationships that could have appeared to influence the work reported in this paper.

Open Access This article is licensed under a Creative Commons Attribution 4.0 International License, which permits use, sharing, adaptation, distribution and reproduction in any medium or format, as long as you give appropriate credit to the original author(s) and the source, provide a link to the Creative Commons licence, and indicate if changes were made. The images or other third party material in this article are included in the article's Creative Commons licence, unless indicated otherwise in a credit line to the material. If material is not included in the article's Creative Commons licence and your intended use is not permitted by statutory regulation or exceeds the permitted use, you will need to obtain permission directly from the copyright holder. To view a copy of this licence, visit http://creativecommons.org/licenses/by/4.0/.

\section{References}

1. Richey RH, Craig JV, Shah UU, Nunn AJ, Turner MA, Barker CE, et al. MODRIC - Manipulation of drugs in children. Int J Pharm. 2013;457:339-41. https://doi.org/10.1016/j.ijpharm.2013.08.061.

2. European Medicines Agency. Guideline on pharmaceutical development of medicines for paediatric use. 2013. https://www. ema.europa.eu/en/documents/scientific-guideline/guidelinepharmaceutical-development-medicines-paediatric-use_en.pdf. Accessed 15 Dec 2021.

3. Akram G, Mullen AB. Mixing medication into foodstuffs: identifying the issues for paediatric nurses. INT J NURS PRACT. 2015;21:125-31. https://doi.org/10.1111/ijn.12222.

4. Martir J, Flanagan T, Mann J, Fotaki N. Recommended strategies for the oral administration of paediatric medicines with food and drinks in the context of their biopharmaceutical properties: a review. J Pharm Pharmacol. 2017;69:384-97. https://doi.org/10. 1111/jphp.12635.

5. Kersten E, Barry A, Klein S. Physicochemical characterisation of fluids and soft foods frequently mixed with oral drug formulations prior to administration to children. Pharmazie. 2016;71:122-7. https://doi.org/10.1691/ph.2016.5145.

6. Martir J, Flanagan T, Mann J, Fotaki N. Impact of Food and Drink Administration Vehicles on Paediatric Formulation Performance: Part 1-Effects on Solubility of Poorly Soluble Drugs. AAPS PharmSciTech. 2020;21:177. https://doi.org/10.1208/s12249-020-01722-z.

7. Manrique YJ, Lee DJ, Islam F, Nissen LM, Cichero JAY, Stokes JR, et al. Crushed tablets: does the administration of food vehicles and thickened fluids to aid medication swallowing alter drug release? J Pharm Pharm Sci. 2014;17:207-19. https://doi.org/10.18433/ j39w3v.

8. Freerks L, Sucher W, Klein S. Relative bioavailability studies with liquids and soft foods to ensure the safe use of dosing vehicles? A critical assessment [Poster presentation at the 11th Conference of the European Paediatric Formulation Initiative (EuPFI), Malmø, Sweden, http://www.eupfi.org/past-conferences/11th-eupfi-conference/].

9. Naumann F, Freerks L, Klein S. "Use of Liquids and/or Soft Foods as Vehicles for Drug Administration": are the vehicles listed in the current FDA draft guidance of global relevance? [Poster presentation at the 12th Conference of the European Paediatric Formulation Initiative (EuPFI), Virtual Conference, http://www.eupfi.org/pastconferences/12th-eupfi-conference-virtual-post/].
10. Food and Drug Administration. Use of Liquids and/or Soft Foods as Vehicles for Drug Administration: General Considerations for Selection and In Vitro Methods for Product Quality Assessments. 2018. https://www.fda.gov/media/114872/download. Accessed 15 Dec 2021.

11. Wollmer E, Klein S. Review of paediatric gastrointestinal physiology relevant to the absorption of orally administered medicines. Adv Drug Deliv Rev. 2022;181: 114084. https://doi.org/10.1016/j.addr. 2021.114084.

12. Wollmer E, Neal G, Whitaker MJ, Margetson D, Klein S. Biorelevant in vitro assessment of dissolution and compatibility properties of a novel paediatric hydrocortisone drug product following exposure of the drug product to child-appropriate administration fluids. Eur J Pharm Biopharm. 2018;133:277-84. https://doi.org/10.1016/j. ejpb.2018.10.022.

13. Wollmer E, Karkossa F, Freerks L, Hetberg A-E, Neal G, Porter J, et al. A Biopredictive In Vitro Approach for Assessing Compatibility of a Novel Pediatric Hydrocortisone Drug Product within Common Pediatric Dosing Vehicles. Pharm Res. 2020;37:203. https://doi.org/ 10.1007/s11095-020-02912-X.

14. Chun AHC, Erdman K, Zhang Y, Achari R, Cavanaugh JH. Effect on bioavailability of admixing the contents of lansoprazole capsules with selected soft foods. Clin Ther. 2000;22:231-6. https://doi.org/ 10.1016/S0149-2918(00)88481-7.

15. Chun AHC, Erdman K, Chiu Y-L, Pilmer BL, Achari R, Cavanaugh $\mathrm{JH}$. Bioavailability of lansoprazole granules administered in juice or soft food compared with the intact capsule formulation. Clin Ther. 2002;24:1322-31. https://doi.org/10.1016/S0149-2918(02) 80036-4.

16. Fay MA, Sheth RD, Gidal BE. Oral absorption kinetics of levetiracetam: The effect of mixing with food or enteral nutrition formulas. Clin Ther. 2005;27:594-8. https://doi.org/10.1016/j.clinthera. 2005.05.010.

17 Pentikis HS, Simmons RDOY, Benedict MF, HatchSJ. Methylphenidate Bioavailability in Adults When an Extended-Release Multiparticulate Formulation Is Administered Sprinkled on Food or as an Intact Capsule. J Am Acad Child Adolesc Psychiatry. 2002;41:4439. https://doi.org/10.1097/00004583-200204000-00017.

18. Eliot L, Butler J, Devane J, Loewen G. Pharmacokinetic evaluation of a sprinkle-dose regimen of a once-daily, extended-release morphine formulation. Clin Ther. 2002;24:260-8. https://doi.org/ 10.1016/S0149-2918(02)85022-6.

19. Thyssen A, Solanki B, Treem W. Randomized, Open-Label, SingleDose, Crossover, Relative Bioavailability Study in Healthy Adults, Comparing the Pharmacokinetics of Rabeprazole Granules Administered Using Soft Food or Infant Formula as Dosing Vehicle Versus Suspension. Clin Ther. 2012;34:1636-45. https://doi.org/10.1016/j. clinthera.2012.06.008

20. Szepes A, Pabst-Ravot A, Storch K, Timpe C. Stability and compatibility of Basmisanil granules co-administered with soft food. Int J Pharm. 2018;553:422-7. https://doi.org/10.1016/j.ijpharm.2018.10. 059.

21. Martir J, Flanagan T, Mann J, Fotaki N. Impact of Food and Drink Administration Vehicles on Paediatric Formulation Performance Part 2: Dissolution of Montelukast Sodium and Mesalazine Formulations. AAPS PharmSciTech. 2020;21:287. https://doi.org/10. 1208/s12249-020-01815-9.

22. Carrier M-N, Garinot O, Vitzling C. Stability and compatibility of tegaserod from crushed tablets mixed in beverages and foods. Am J Health-Syst Ph. 2004;61:1135-42. https://doi.org/10.1093/ajhp/61. 11.1135 .

23. Wells KA, Losin WG. In vitro stability, potency, and dissolution of duloxetine enteric-coated pellets after exposure to applesauce, apple juice, and chocolate pudding. Clin Ther. 2008;30:1300-8. https:// doi.org/10.1016/s0149-2918(08)80054-9. 
24 Klein S, Butler J, Hempenstall JM, Reppas C, Dressman JB. Media to simulate the postprandial stomach I. Matching the physicochemical characteristics of standard breakfasts. J Pharm Pharmacol. 2004;56:605-10. https://doi.org/10.1211/0022357023367.

25. Pink Lady ${ }^{\circledR}$ apple. Apple nutrition. https://www.pinkladyapples.co. uk/the-crunch/pink-lady-news/apple-nutrition-vitamins-and-miner als. Accessed 2 Mar 2021.

26. Batchelor HK. Influence of Food on Paediatric Gastrointestinal Drug Absorption Following Oral Administration: A Review. Children. 2015;2:244-71. https://doi.org/10.3390/children2020244.

27. Kofoed-Djursner C, Jamil A, Selen A, Müllertz A, Berthelsen R. Drug solubilization during simulated pediatric gastro-intestinal digestion. Eur J Pharm Sci. 2021;162: 105828. https://doi.org/10. 1016/j.ejps.2021.105828.

28. Boyd BJ, Salim M, Clulow AJ, Ramirez G, Pham AC, Hawley A. The impact of digestion is essential to the understanding of milk as a drug delivery system for poorly water soluble drugs. J Control Release. 2018;292:13-7. https://doi.org/10.1016/j.jconrel.2018.10.027.

29. Marciani L, Gowland PA, Spiller RC, Manoj P, Moore RJ, Young $\mathrm{P}$, et al. Effect of meal viscosity and nutrients on satiety, intragastric dilution, and emptying assessed by MRI. Am J Physiol Gastrointestinal Liver Physiol. 2001;280(6):G1227-33. https://doi.org/10.1152/ ajpgi.2001.280.6.G1227.
30. Shimoyama Y, Kusano M, Kawamura O, Zai H, Kuribayashi S, Higuchi T, et al. High-viscosity liquid meal accelerates gastric emptying. Neurogastroenterol Motil. 2007;19:879-86. https://doi.org/10. 1111/j.1365-2982.2007.00972.x.

31. Ménard O, Famelart MH, Deglaire A, Le Gouar Y, Guérin S, Malbert $\mathrm{CH}$, et al. Gastric Emptying and Dynamic In Vitro Digestion of Drinkable Yogurts: Effect of Viscosity and Composition. Nutrients. 2018 Sep 14;10(9):1308. https://doi.org/10.3390/nu10091308.

32. Liu W, Jin Y, Wilde PJ, Hou Y, Wang Y, Han J. Mechanisms, physiology, and recent research progress of gastric emptying. Crit Rev Food Sci Nutr. 2021;61(16):2742-55. https://doi.org/10.1080/10408 398.2020.1784841.

33. Food and Drug Administration. Assessing the Effects of Food on Drugs in INDs and NDAs - Clinical Pharmacology Considerations. 2019. https://www.fda.gov/regulatory-information/search-fda-guida nce-documents/assessing-effects-food-drugs-inds-and-ndas-clinicalpharmacology-considerations. Accessed 15 Dec 2021.

Publisher's Note Springer Nature remains neutral with regard to jurisdictional claims in published maps and institutional affiliations. 